\title{
I nitial Rendezvous Protocol using Multicarrier Operation for Cognitive Radio Ad-hoc Networks
}

\author{
Ik-Soo Choi ${ }^{1}$, Sang-Jo Yoo ${ }^{1}$, Myunghwan Seo ${ }^{2}$, Chul-Hee Han' and Bongsoo Roh ${ }^{3}$ \\ ${ }^{1}$ Department of Information and Communication Engineering, University of Inha \\ Incheon, Korea \\ [e-mail: ischoi.win@gmail.com, sjyoo@inha.ac.kr] \\ ${ }^{2}$ Hanwha Systems \\ Daejeon, Korea \\ [e-mail: myunghwan.seo@hanwha.com, chulhee.han@hanwha.com] \\ ${ }^{3}$ The $2^{\text {nd }} \mathrm{R} \& D$ Institute, Agency for Defense Development \\ Daejeon, Korea \\ [e-mail: saintroh@add.re.kr] \\ *Corresponding author: Sang-Jo Yoo
}

Received October 31, 2017; revised January 9, 2018; accepted February 5, 2018;

published June 30, 2018

\begin{abstract}
In cognitive radio technology, the overall efficiency of communications systems can be improved without allocating additional bands by allowing a secondary system to utilize the licensed band when the primary system, which has the right to use the band, does not use it. In this paper, we propose a fast and reliable common channel initialization protocol without any exchange of initialization messages between the cluster head and the member nodes in cognitive ad-hoc networks. In the proposed method, the cluster and member nodes perform channel-based spectrum sensing. After sensing, the cluster head transmits a system activation signal through its available channels with a predetermined angle difference pattern. To detect the cluster head's transmission channels and to join the cluster, each member node implements fast Fourier transform (FFT) and computes autocorrelation for the angle difference sequence of the received signal patterns. This is compared to the predetermined reference angle difference pattern. The join-request and channel-decision procedures are presented in this paper. Performance evaluation of the proposed method is presented in the simulation results.
\end{abstract}

Keywords: Cognitive radio networks, common channel, average angle difference pattern, system activation signal, boundary symbol synchronization.

A preliminary version of this paper appeared in ICTC 2016, October 19-21, Jeju, Korea. This version includes a concrete analysis and supporting implementation results on initial rendezvous protocol using multicarrier operation. This research was supported by a grant-in-aid of Hanwha Systems and the Agency for Defense Development (ADD) in the Republic of Korea as part of the Contract UC 160007ED. 


\section{Introduction}

In recent years, developments in several communications systems and devices have led to scarcity of spectrum, especially in defense and military applications. Moreover, interference is increasing among the communications devices in narrow operation areas. Therefore, they require efficient management methods for frequency resources. According to the Federal Communications Commission (FCC), the United States will run out of radio spectrum in the upcoming years [1]. The spectrum regulatory bodies in different countries assign spectrum to licensed users, also termed primary users (PUs), on a long-term, persistent basis for large geographic regions. However, a large portion of the assigned spectrum remains underutilized [2]. Therefore, in order to improve utilization of the spectrum, with an aim to address the critical problem of spectrum scarcity, the possibility of opening licensed frequency bands to unlicensed operations has been considered [3-5]. Generally, licensed users, or PUs, have the right to utilize the spectrum without interference. However, unlicensed users, also known as secondary users (SUs), are allowed to opportunistically exploit unused licensed spectrum, called spectrum holes, to send data. If a PU is currently not using its spectrum, the SU changes its transmission parameters to take advantage of the spectrum hole. After discovering the unoccupied frequency bands, an SU selects the best available channel for opportunistic communications. The SU must vacate the channel when the PU returns.

Cognitive radio (CR) devices equipped with a dynamic spectrum access capability are able to detect spectrum opportunities in the licensed bands and map them into logical channels, which can be temporarily used by the CR nodes (secondary users) for communications. Therefore, CR is regarded as a promising technology to cope with the spectrum scarcity problem. In CR networks, unlicensed users are allowed to use the temporarily unused licensed bands as long as they do not interfere with the communications of the licensed users [6]. One of the key concerns in using CR technology is to guarantee that PU transmissions are always secured. To achieve this, it is vital to exchange information pertaining to the spectrum availability between a given node pair before starting data transmission.

The methods to successfully negotiate common channels among SUs have been taken into consideration [7] and can be classified into sequence-based methods and group-based methods. In a sequence-based method, each node changes its negotiating channel in accordance with a random or a predetermined channel-hopping sequence [8]. Sequence-based methods use a common channel that is selected from a hopping pattern [9], in which the mechanism to decrease rendezvous-setting time is used in a hop-and-wait channel hopping scheme. In literature, the rendezvous-setting time represents the required time to setup an initial common channel between cluster head and member nodes. The important issue is how quickly member nodes find the operating channels (or signals) of the cluster head. Group-based methods manage control messages based on a common channel that is negotiated by each node [10], in which for multi-hop ad-hoc networks, a multicasting, tree-based initial common channel method was proposed. In general, a group-based method obtains common channel coverage better than a sequence-based method, and if a specific group has many nodes in a secondary group, then it causes a channel saturation problem.

In many cluster-based CR ad-hoc networks, such as wireless sensor networks and group-based device-to-device networks, in which a cluster head can communicate directly with its member nodes, the cluster head needs to determine the common channel according to the results of spectrum sensing $[10,11]$. In this paper, we propose a new common 
channel-setting protocol for cluster-based CR networks. In CR wireless networks, each node may have different available channel sets, because they sense different channels according to their location. In military CR networks especially, each node has a different channel set because the distance between each member node is from hundreds of meters up to several kilometers. Member nodes have to transfer information about sensing results to the cluster head to join the cluster. So, the common channel initial process is handled reliably and quickly by the cluster head.

In the proposed method, the cluster head transmits to member nodes a system activation (SA) signal that has a specific pattern before exchanging the control messages between itself and member nodes. The SA signal sequence is designed to have a specific angle difference pattern. Member nodes perform fast Fourier transform (FFT) on their available channels to precisely detect the SA signal sequence transmitted by the cluster head. Then, member nodes calculate the autocorrelation using a correlation-based matching method. After this, the process of joining the cluster is started. The cluster head determines the common channel or channels for the group.

We propose a reliable common channel method using orthogonal frequency division multiplexing (OFDM)-based multicarrier operation and common pattern-sensing schemes to determine the common channel by the cluster head. The process of determining the common channel is designed in three stages. First is system activation, followed by a reporting period; the last stage is data transmission.

The rest of this paper is organized as follows. In Section II, the summary of the related work is presented. In Section III, the proposed secondary system activation mechanism is presented. In Section IV, the member node-join and cluster head's common channel-decision procedures are explained. In Section V, the performance of the proposed method is evaluated, and finally, Section VI concludes this paper.

\section{Related Work and New Approach}

In this section, we introduce the conventional common-channel setup algorithms used for communications in a ad-hoc CR network. We also analyze the disadvantages of the conventional methods, and explain the new schemes of the proposed method.

Rendezvous protocol in cognitive radio networks represents the process by which secondary users establish links on a common available channel between themselves before data transmission. Rendezvous protocols can be classified into common control channel based protocol and channel hopping based rendezvous protocol [12].

In common control channel based protocol, it is generally assumed that a global common control channel is available to all CR nodes in the network for exchanging channel negotiation control messages to determine a data channel. In fact this assumption is not sometimes realistic in the practical world [13].

For channel hopping based protocol, in [14], Quorum-based Channel Hopping (QCH) system is proposed that is robust against link breakage caused by the appearance of incumbent user signals. The Quorum and Latin square Channel Hopping (QLCH) protocol is proposed in [15], where the time slots for secondary users are divided into default and switching slots. In [16], an efficient channel hopping protocol called randomized QLCH protocol (RQL) is proposed. Nodes running RQL may change their original channel hopping sequences dynamically according to their intended receivers. However, in the both schemes, the secondary users need to know all the identifications and the time slot offsets of their neighbor secondary users. Asynchronous Quorum-based blind rendezvous channel hopping algorithm 
is presented in [17]. In [18], to decrease rendezvous time and increase network throughput, a channel hopping protocol using Hadamard matrix and hash function is proposed. [19] presents a systematic approach for designing channel hopping protocols for CR networks that maximize rendezvous diversity between any two nodes even if their clocks are asynchronous. [13] surveys existing work on channel hopping based rendezvous algorithms and compares their performances in terms of the maximum time to rendezvous. According to the analysis of [13], the maximum time to rendezvous of the most of asynchronous rendezvous algorithms is $O\left(N^{2}\right)$ slot time, in which $N$ is the number of channels.

Most of channel hopping based algorithms are applied to two CR nodes so that there is a limitation on broadcasting or multicasting packet delivery in cluster-based ad-hoc networks. In cluster-based ad-hoc network, cluster head invites member nodes and member nodes join the cluster by detecting cluster head signal $[10,11,20,21]$. By determining a common data channel for the cluster, each node can easily send and receive any types of traffic. The resource allocation between member nodes can be done by cluster head. In the conventional cluater-based methods, the cluster head sequentially and repeatedly broadcasts the SA message, which carries all available channel information for the cluster head. Member nodes listen to one of the available channels for a predetermined time. If member nodes do not receive the SA message from the cluster head, they listen to the next available channel. After member nodes decode the received SA message, they report the message, including their available channel information, in the reporting period. The cluster head determines the common data channel using the received information and broadcasts the information about the selected common data channels on the selected channels. At this moment, the message is called a channel decision (CD) message, which has the selected channel information and the member nodes list. Once the member nodes successfully receive the CD message, they can transmit data using the selected channel.

The problem in the conventional cluster-based (group-based) rendzvous methods is that it requires a long time to successfully receive the SA message. When the total number of channels is $N$ and SA transmission time is $T_{S A}$ on each channel, the cluster head transmits SA message on each channel with round robin manner. Each member node selects one channel from its available channels and waits a CH's SA message at least $N \times T_{S A}$ time. If a member node does not receive SA message on the channel, then it switches to the next channel and waits again. It is performed for $N$ channels at most so that the maximum rendezvous time becomes $N \times N \times$ $T_{S A}$. Furthermore, if the total number of channel increases, SA signal transmission time also increases, because the cluster head has to transmit the SA signal on all available channels.

In another rendezvous method, the cluster head selects a single channel using the local sensing result, and starts the joining process on the chosen channel. Member nodes can join the cluster group after they detect the cluster head's system channel. To sense a secondary cluster head, cyclostationary signal sensing is used. However, if the cluster head does not have the member nodes' available channels, some of the member nodes may not be able to listen to the current operating channel chosen by the cluster head. Thus, such member nodes cannot join the system.

To solve these problems, we propose a new approach to transmitting the SA signal using orthogonal frequency division multiplexing (OFDM)-based multichannel operation. We assume that a wide band is divided into channels, in which a channel (or channels) will be used for the CR ad-hoc system. The chosen channel will also be sub-divided into multiple subcarriers for CR OFDM system operation. Using all the available channels of the cluster head, rendezvous pattern OFDM signals are transmitted. Other non-available channels are deactivated. An available channel may have several sub-channels (subcarriers), and some 
boundary sub-channels are also deactivated to reduce possible harmful interference. The available channel (or sub-channel) carries pattern values. One feature of the pattern signals is defined as the angle difference of the consecutive pattern signals. Each member node performs multi-channel decoding. Using the OFDM decoding mechanism, member nodes decode multi-channel pattern signals to find the channels on which the rendezvous pattern exists. The time correlation of the pattern from the observed pattern signals on each channel is compared with the reference correlation. Pattern signal decoding is only performed for the available channels of each member node. The correlation matching value is evaluated to determine the reporting channel of a member node.

A preliminary version of this paper appeared in [21], in which we proposed a common channel setup method in the ad-hoc cognitive radio networks using multicarrier operation. In [21], we differentiated the cluster groups with different angle difference patterns. Using multi-channel OFDM technology, member nodes can quickly perform system activation signal detection that is sent from the cluster head. In this paper, we have included new additional protocol and system features for reliable initial rendezvous operation in ad-hoc cognitive radio networks. In this version, we have extended the angle difference pattern derivation not only with single activated subcarrier but also with multiple activated subcarriers to reduce the rendezvous time. We also have added synchronization frame format and rendezvous protocol procedures between the cluster head and member nodes to precisely explain the required system behaviors. More comprehensive analysis and simulation results are also presented in this paper.

The proposed method has some advantages, compared to the conventional methods. We have a fast rendezvous time due to use of multi-channel OFDM pattern signal transmission and concurrent detection of cluster head patterns on multiple channels. The proposed rendezvous protocol operates asynchronous mode without global clock synchronization. Between the cluster head and member nodes, without initial synchronization, the pattern feature of the cluster head can be easily found using the proposed boundary detection mechanism. The defined pattern feature (angle difference) is strong against OFDM symbol synchronization mismatch and slow fading. The differentiation of clusters uses a different pattern signal feature, and member nodes can easily recognize the desired cluster group to join from among multiple cluster groups. The pattern signal transceiver uses the OFDM architecture. Data transmission also uses the same OFDM system. Only the subcarrier bandwidth is different. The notations used throughout this paper are summarized in Table $\mathbf{1}$.

Table 1. Notations used in this paper

\begin{tabular}{|c|c|}
\hline Notations & Descriptions \\
\hline \hline$N$ & Number of channels \\
\hline$N$ & Number of subcarriers of a channel \\
\hline$L$ & Number of boundary subcarriers \\
\hline$P S Q$ & Pattern signals with length \\
\hline$A Q$ & SA pattern signal sequence \\
\hline$A D Q$ & Angle sequence \\
\hline$R_{i}^{j}[t]$ & Angle difference sequence \\
\hline$R_{r}[t]$ & Autocorrelation of the observed pattern for channel $j$ at member \\
& node $i$ \\
\hline$R_{i, j}$ & Autocorrelation of the reference pattern \\
\hline & Correlation with $i$-th and $j$-th symbols \\
\hline
\end{tabular}




\section{System Activation Mechanism}

\subsection{Network Topology}

As shown in Fig. 1, in the proposed method, we assume that a cluster head and several member nodes exist within bi-directional communications range. If a secondary node is inside the primary signal sensing range of a primary system, then it can detect the primary signal. Cluster head and member nodes may have different available channel sets as a result of each node's spectrum sensing. There is no common channel to exchange control information.

Fig. 2 illustrates the sensing model. In Fig. 2, a sensing threshold is determined to guarantee that sensing range and sensing threshold are determined to guarantee that any transmission from a secondary node that is located outside the primary sensing range does not cause any harmful interference on the primary system. A channel is available when the primary signal power is less than a sensing threshold, as determined in (1):

sensing threshold $\leq P_{S T}-P L(d 1+d 2+d 3)$

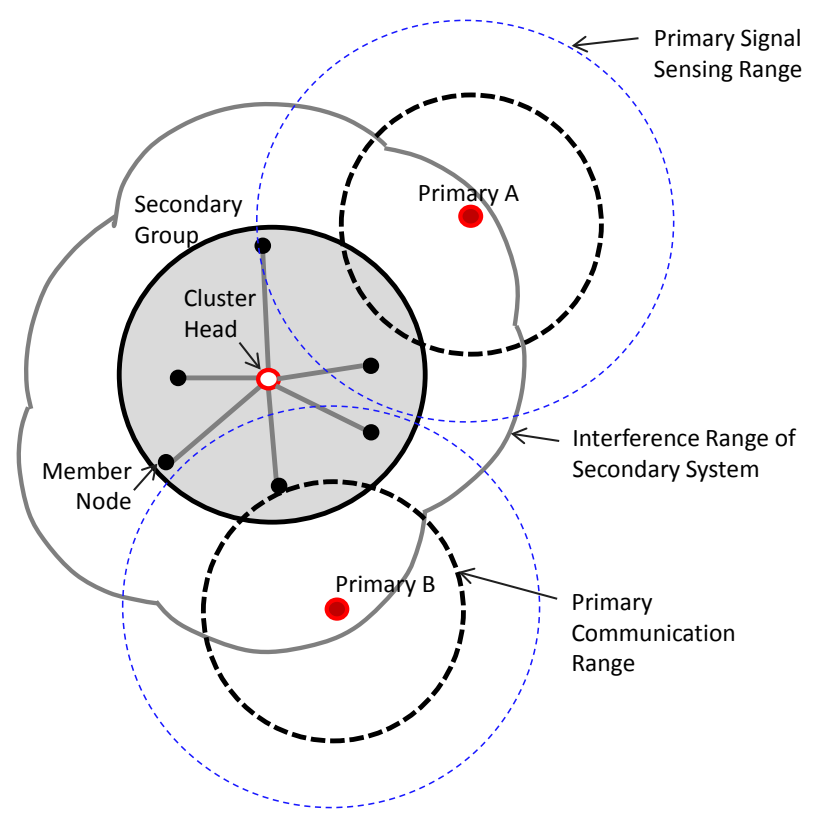

Fig. 1. Cognitive radio ad-hoc networks.

\subsection{Multicarrier Operation Model}

For the initial setup (rendezvous period), wideband multicarrier modulation is used. A subcarrier for transmitting the SA signal is relatively wider than that of data transmission. In the actual communication phase, subcarrier spacing will be narrowed. 


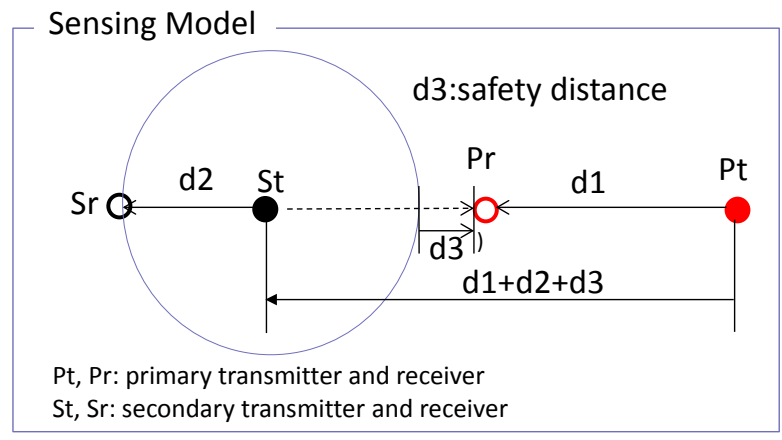

Fig. 2. Sensing model.

Basic channel bandwidth that a secondary system can utilize is determined in advance. The entire band of interest is sub-divided into multiple channels. A secondary system will use one or more consecutive channels for data communications. For spectrum sensing, the wide band is subdivided into $N$ channels. Each node performs channel-based spectrum sensing. The cluster head and member nodes obtain their local available channel set. During the initial rendezvous period, a channel has $M(\geq 1)$ subcarriers. The total number of subcarriers for initial setup is (Number of channels) $\times M=$ Total subcarriers for the rendezvous period. At each channel boundary, a subcarrier is located. Therefore $(M-1)$ subcarriers exist within a channel. In the proposed model, frequency offset is not critical because the subcarrier spacing is relatively wide.

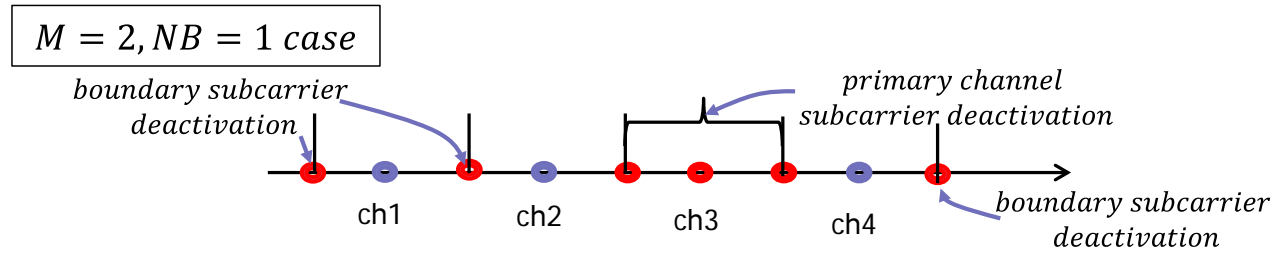

(a) The states of subcarriers when $M=2$ and $N B=1$.

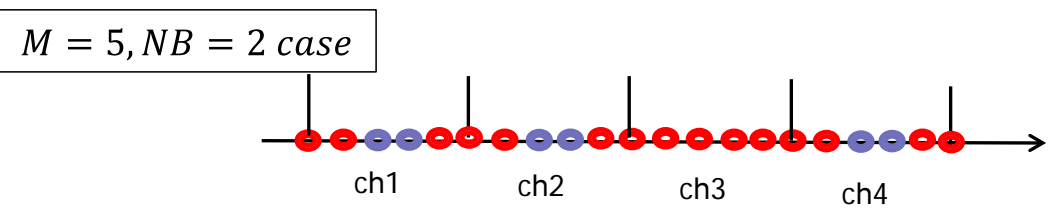

(b) The states of subcarriers when $M=5$ and $N B=2$.

Fig. 3. States of subcarriers.

We propose subcarrier deactivation rules where all subcarriers of the primary detected channels are deactivated by the cluster head to avoid any harmful interference to primary systems. The predefined number of boundary subcarriers $(N B)$ is also deactivated to reduce OFDM side-lobe interference with other SA signals. Fig. 3 shows the states of subcarriers according to case. In Fig. 3, blue circles represent the activated subcarriers, and red circles represent the deactivated subcarriers. 
SA signals are transmitted on all the activated subcarriers of the cluster head's available channels using OFDM. SA signal sequence is designed to have a specific pattern. The SA signal (pattern signal) sequence in the time domain is input for OFDM. The same pattern signal sequence is applied to all available channels of the cluster head. Each cluster head has different pattern signal sequences. In Fig. 4, we can see that pattern signal sequence is transmitted on all subcarriers that have the specific pattern. All activated subcarriers of each available channel carry SA pattern signal input. At a given time, the input set for $N$-point inverse fast Fourier transform (IFFT) is converted into an OFDM symbol.

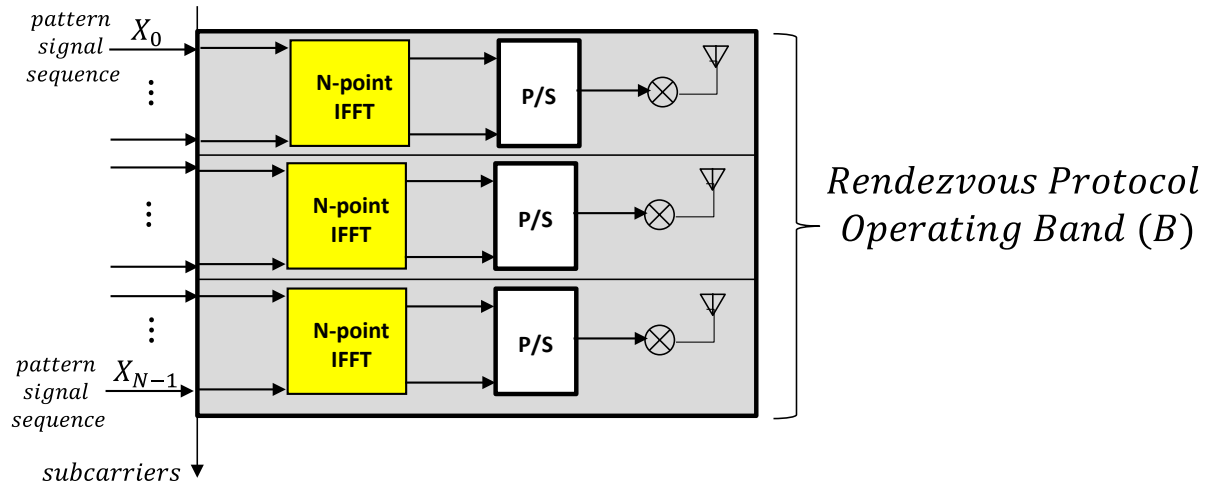

Fig. 4. Multi-band OFDM system.

\subsection{Angle Difference Derivation Rules}

The pattern signal sequence is predetermined for each cluster group. Each cluster head has its own pattern signal sequences, which are given to member nodes as well. A pattern signal is a complex value, and a pattern sequence is a set of pattern signals with length $L$.

Patterns should be derived easily and robustly by each member node without any timing synchronization and channel estimation processes by member nodes. Member nodes may start decoding SA OFDM symbols at any time, not from the actual symbol boundary. Member nodes can experience different signal attenuations.

In this paper, we define the pattern as an angle difference sequence. From the pattern signal sequence, the angle difference sequence is obtained. Angle is robust to signal attenuation and phase shift caused by time delay in the OFDM system.

SA pattern signal sequence (PSQ) of a cluster head $i$ can be represented with (2).

$$
P S Q_{i}=\left\{c_{1}, c_{2}, \cdots, c_{L}\right\}
$$

where $c_{k}$ is $k$ th complex value pattern signal (i.e. $c_{k}=a_{k}+j b_{k}$ ).

From $P S Q_{i}$, we can calculate the angle sequence $\left(A Q_{i}\right)$ in polar coordinates. We count clockwise angles such that angle range $=[-\pi,+\pi]$, as seen in (3).

$$
A Q_{i}=\left\{\angle c_{1}, \angle c_{2}, \cdots, \angle c_{L}\right\}
$$

From $A Q_{i}$, the predefined angle difference sequence $\left(A D Q_{i}\right)$ derivation rules are applied, depending on the number of activated subcarriers for each available channel. If only a single activated subcarrier for a channel exists, then $A D Q_{i}$ is given by 


$$
A D Q_{i}=\left\{p_{1}, p_{2}, \cdots, p_{L-1}\right\}=\left\{\left(\angle c_{2}-\angle c_{1}\right),\left(\angle c_{3}-\angle c_{2}\right), \cdots,\left(\angle c_{L}-\angle c_{L-1}\right)\right\}
$$

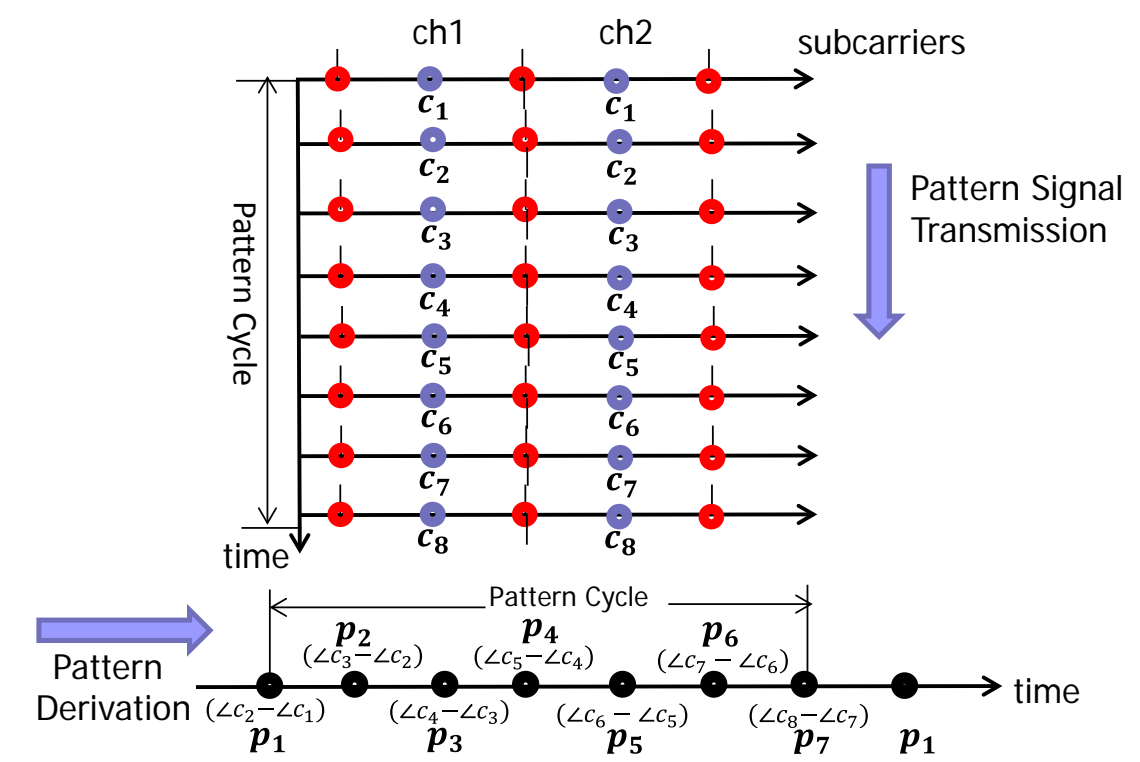

Fig. 5. Angle difference pattern derivation with only one activated subcarrier.
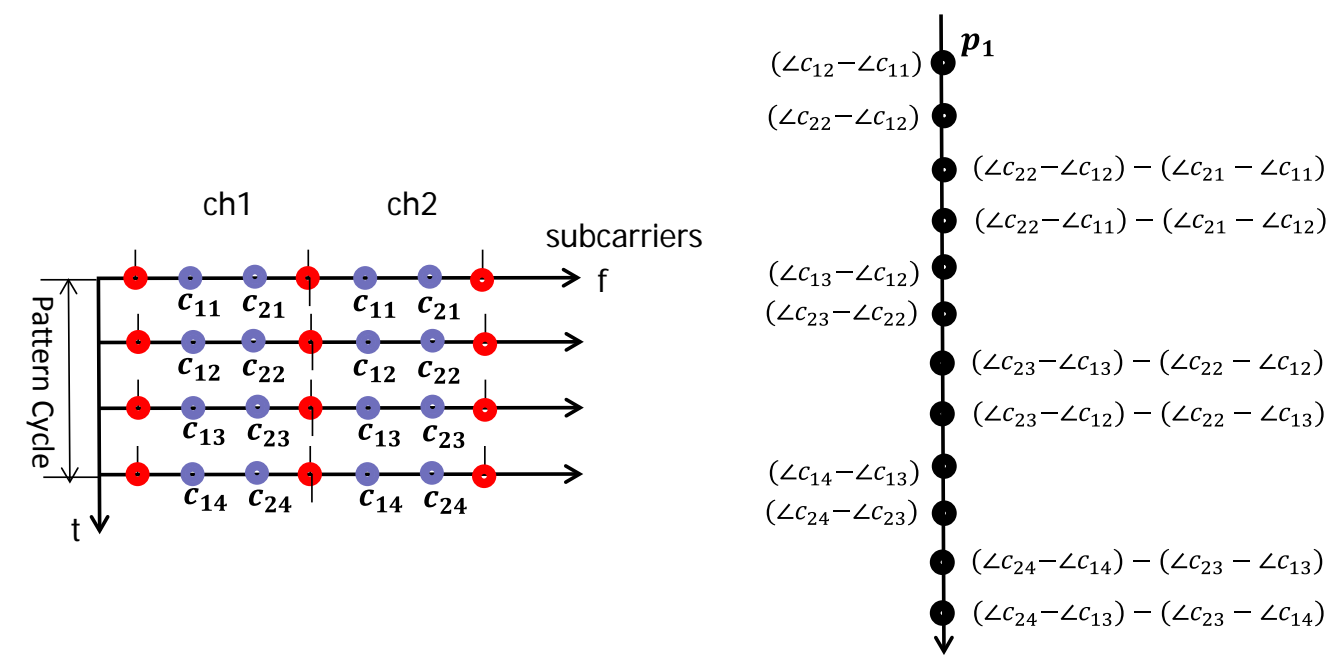

Fig. 6. Angle difference pattern derivation with multiple activated subcarrier.

Fig. 5 illustrates an example of deriving the angle difference pattern in case each available channel has only one activated subcarrier. The pattern for the cluster head is defined as $A D Q_{i}$ with length (L-1). In Fig. 6, each available channel has multiple activated subcarriers. Depending on the number of activated subcarriers, different angle difference derivation rules can be made. We can use not only difference sequence for each subcarrier, but also between subcarriers. 


\subsection{System Activation (SA) Pattern Matching Mechanism}

In this paper, we use the pattern-matching method for a correlation-based pattern matching method. At each member node, for every available channel of the member node, the decoded angle difference sequence is compared with the reference pattern. For the angle difference sequence of the received pattern signals, autocorrelation is computed then compared with the autocorrelation of the reference (ideal) angle difference pattern sequence. If the matching result is higher than a threshold (or lower, depending on the matching mechanism), then the matched channel indicates that the cluster head has transmitted the SA pattern signals. It means that the channel is one of the common available channels between the cluster head and the member nodes. Two correlation matching methods are proposed.

The first matching method is product-based similarity matching, as defined with (5).

$$
S \_P_{i}^{j}=R_{i}^{j} * R_{r}=\frac{1}{T} \sum_{t=0}^{T-1} R_{i}^{j}[t] R_{r}[t]
$$

where,

$R_{i}^{j}[t]=$ autocorrelation observed pattern for channel $j$ at member node $i$ with time lag $t$

$R_{r}[t]=$ autocorrelation of the reference pattern

$T=$ number of autocorrelation samples

$S \_P_{i}^{j}$ is compared with a threshold value. If $S_{-} P_{i}^{j} \geq T h_{s p}$, then the member node considers the SA signal to be sent on channel $j$.

The second matching method is mean square error-based similarity matching as in (6):

$$
S \_M S E_{i}^{j}=E\left[\left(R_{i}^{j}[t]-R_{r}[t]\right)^{2}\right]=\frac{1}{T} \sum_{t=0}^{T-1}\left(R_{i}^{j}[t]-R_{r}[t]\right)^{2}
$$

where if $S_{-} M S E_{i}^{j} \leq T h_{s m s e}$, then the member node considers the SA signal to be sent on channel $j$.

\subsection{System Activation (SA) Pattern Matching Mechanism}

In the conventional OFDM systems, the set of subcarriers (activated) is predetermined on the operating band. We define a pattern symbol that has a special symbol in the time domain (i.e. predetermined IFFT input values for all subcarriers). The receiver can compare the received symbol value with the reference symbol pattern using correlation, and adjusts the received symbol boundary with a time shift. The time offset that has the maximum correlation value is selected. In the CR environment, this mechanism cannot be applied, because the activated subcarrier set is dynamic in time and location, depending on the neighboring primary system status. In addition, each member node's available channel set (set of decoding subcarriers) is different. Therefore, we cannot determine a pattern symbol value.

We assume that cluster head and member nodes may not be synchronized. If the OFDM symbol start time is not synchronized, then it results in constant phase shift when a duplicated OFDM symbol follows. For the next pattern signal, the same phase shift can be assumed, because OFDM symbol duration is fixed and initial decoding delay is kept for consecutive pattern signals. Therefore, pattern generation using the angle difference between pattern signals can remove the phase shift effect due to the OFDM symbol detection time difference. 
Fig. 7 shows that one OFDM symbol that carries subcarrier pattern signals is repeated $Q$ $(\geq 3)$ times.

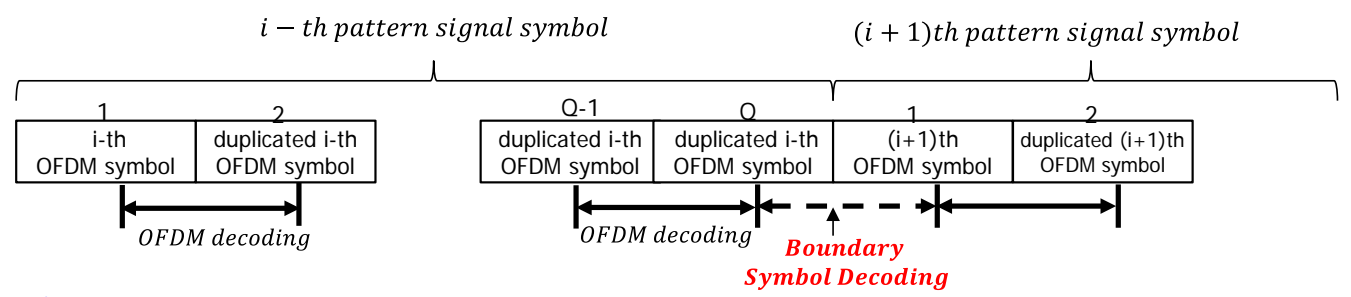

Fig. 7. Member node performs decoding between OFDM symbol and duplicated OFDM symbol.

If the conditions given in (7) are satisfied, then the decoded $n$-th OFDM symbol is considered a boundary symbol:

$$
\left\{\begin{aligned}
R_{n, n-1}<R_{t}, & R_{n, n+1}<R_{t} \\
R_{n-1, n-2}>R_{t}, & R_{n+1, n+2}>R_{t}
\end{aligned}\right.
$$

where, $R_{i, j}$ is the correlation with $i$-th and $j$-th symbols and $R_{t}$ is the correlation threshold.

When we compute the angle for the i-th received pattern signal for each subcarrier, the average of the angles of the pattern signals is taken using the duplicated symbols, except the boundary one. Once a member node detects the cluster head's pattern, it can perform symbol boundary synchronization, as shown in Fig. 8. The member node adjusts the boundary point around the boundary symbol and finds the best time-offset that shows maximum symbol correlation with the previous or next symbols.

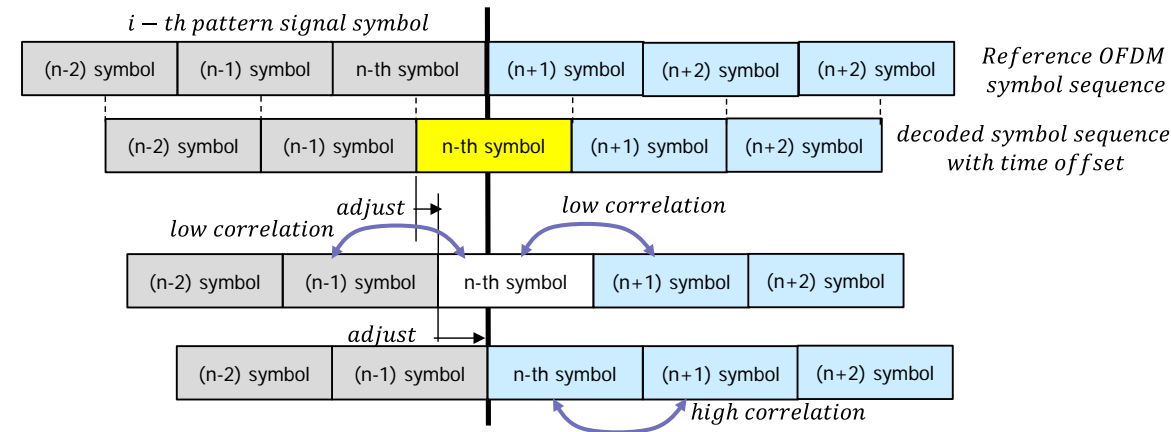

Fig. 8. Boundary symbol synchronization.

To sum up the system activation mechanism in Fig. 9, the cluster head transmits the SA signal, which has a specific angle difference pattern, to the activated subcarriers. Member nodes perform OFDM symbol decoding (FFT) on their own available channels, and distinguish the angle difference pattern. If member nodes successfully recognize the angle difference pattern on the channels, then the cluster head and member nodes begin the reporting period. 


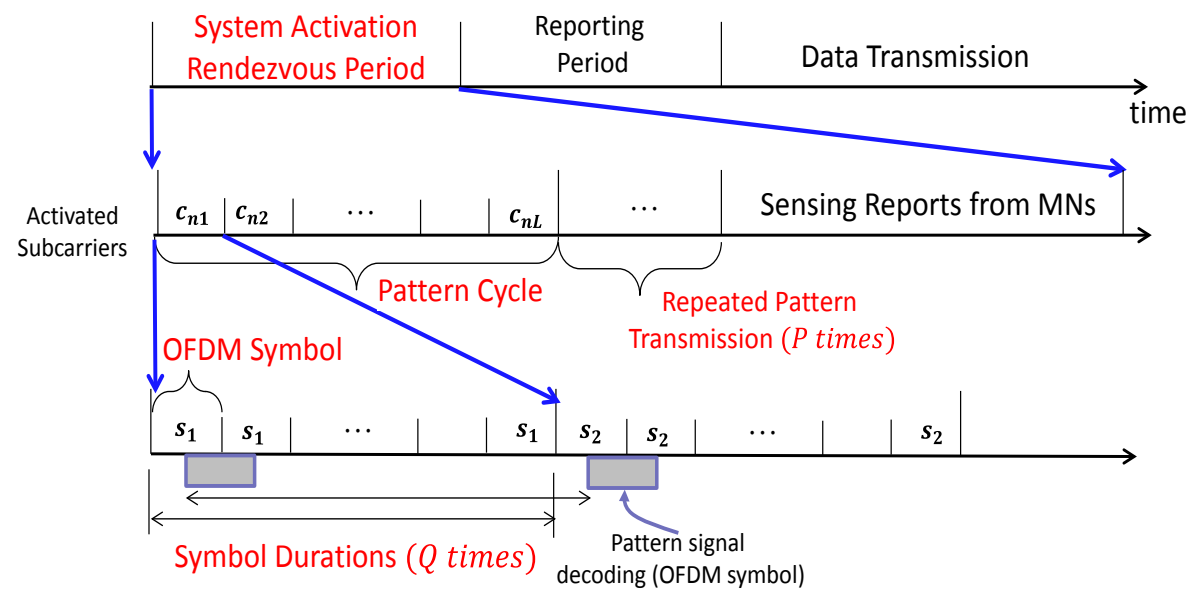

Fig. 9. System activation rendezvous period procedure.

\section{Cluster Join and Common Channel Decision Notification Procedure}

After a predetermined amount of pattern broadcasting, the cluster head starts the reporting period using its available channels. The cluster head sends a reporting start (RS) message on all activated subcarriers of the available channels. During the reporting period, member nodes report their available channel lists. Member nodes listen to one of the subcarriers for which the SA pattern was matched. If multiple matched subcarriers (=S) exist, one of the subcarriers is selected randomly with probability $=1 / \mathrm{S}$. If a member node receives an RS message, it replies with a channel report (CR) message that includes the member node's available channels. The cluster head then decides on the data channel(s) to use based on the member node's reports. The cluster head broadcasts the channel decision (CD) message on all subcarriers from which CR messages are received.

The secondary system operates only on the selected channel(s) for normal data transmission. To allow new nodes to join, the cluster head periodically performs the initial setup procedure. The periodic initial setup procedure can be performed on all the available channels of the cluster head or only on the currently used data channel(s). Fig. 10 illustrates the procedure for determining common data channel(s).

We propose a common data initial method using the SA signal, which has a specific angle difference pattern in combining an OFDM system and dynamic spectrum access (DSA) technology. In Fig. 11-(a), we can see that a DSA structure is added to the sender (cluster head) designed for the primary signal detector that can detect primary systems. The boundary subcarrier deactivation rule maker deactivates the boundary subcarriers, and the initialization pattern generator makes the angle difference pattern. In Fig. 11-(b), the receiver calculates autocorrelation compared to the received signal and reference signal. If the receiver successfully recognizes the pattern signal, then it starts the process of setting the common data channel. 


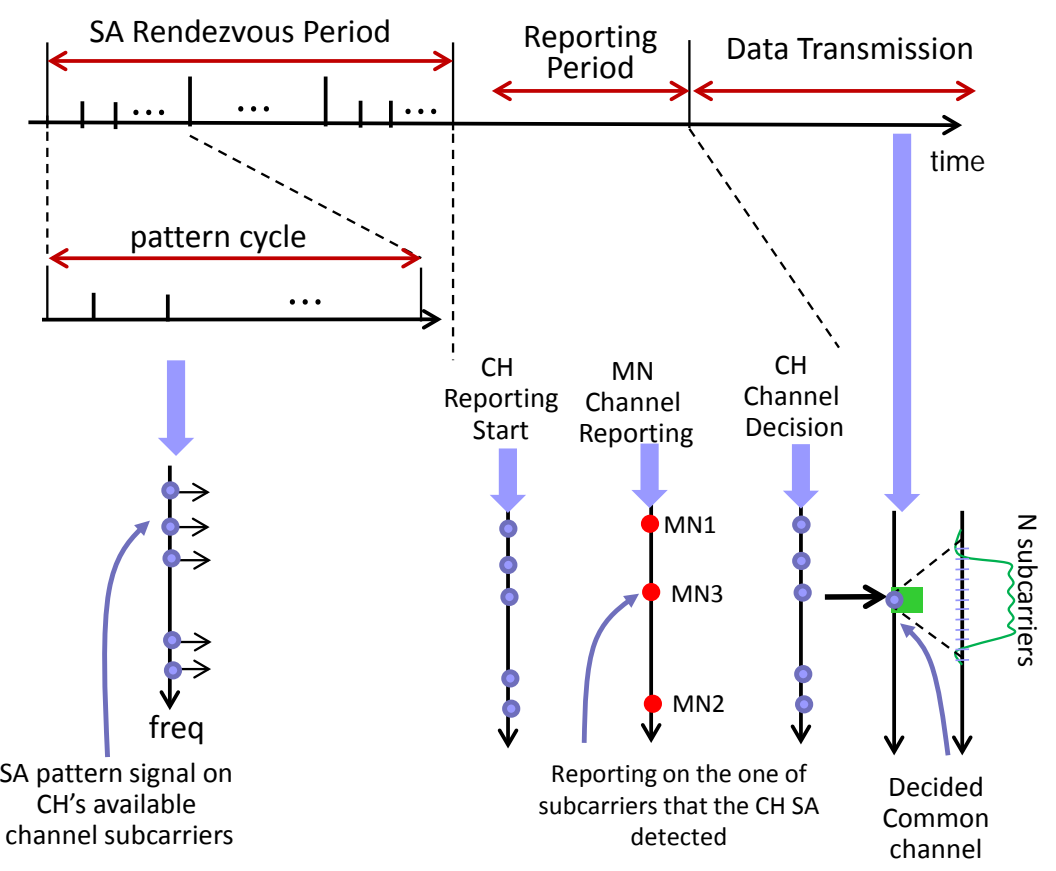

Fig. 10. The procedure for determining the common data channel(s).

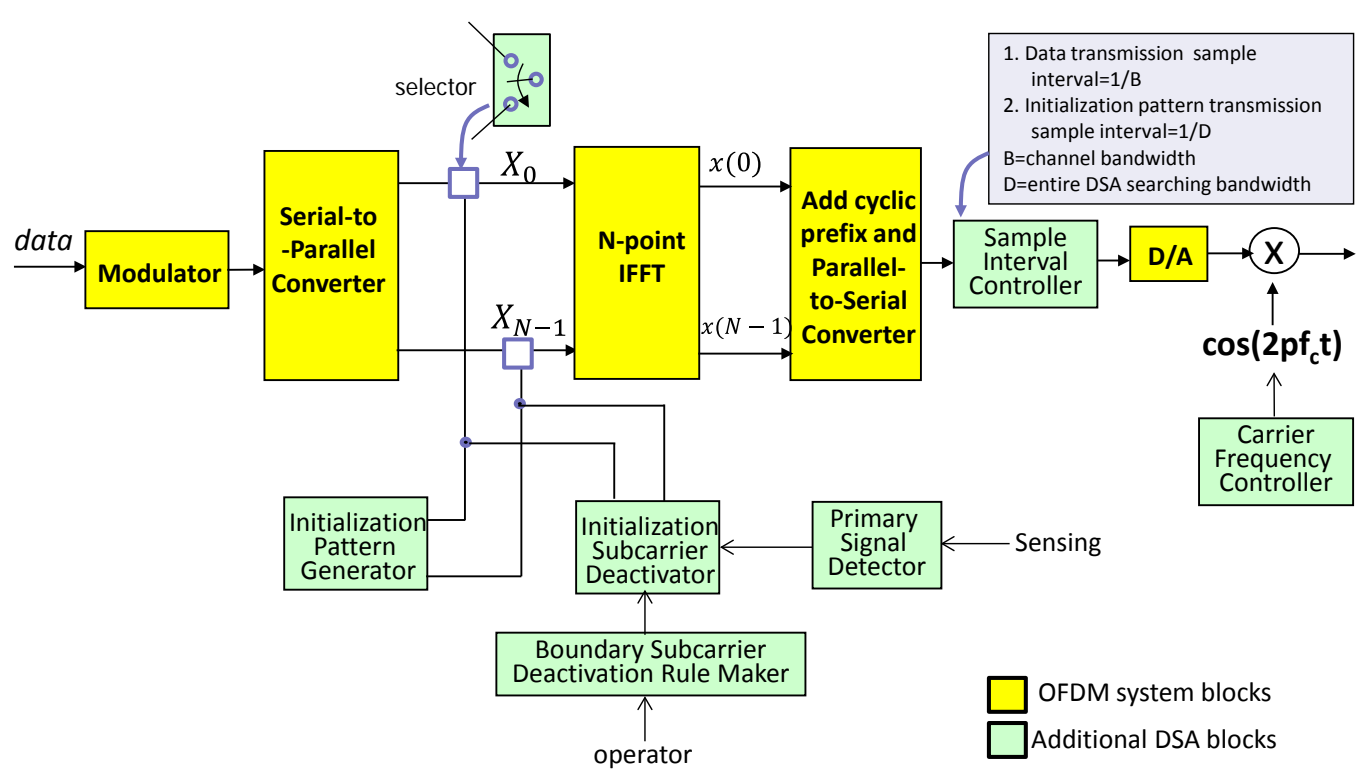

(a) System architecture of the transmitter. 


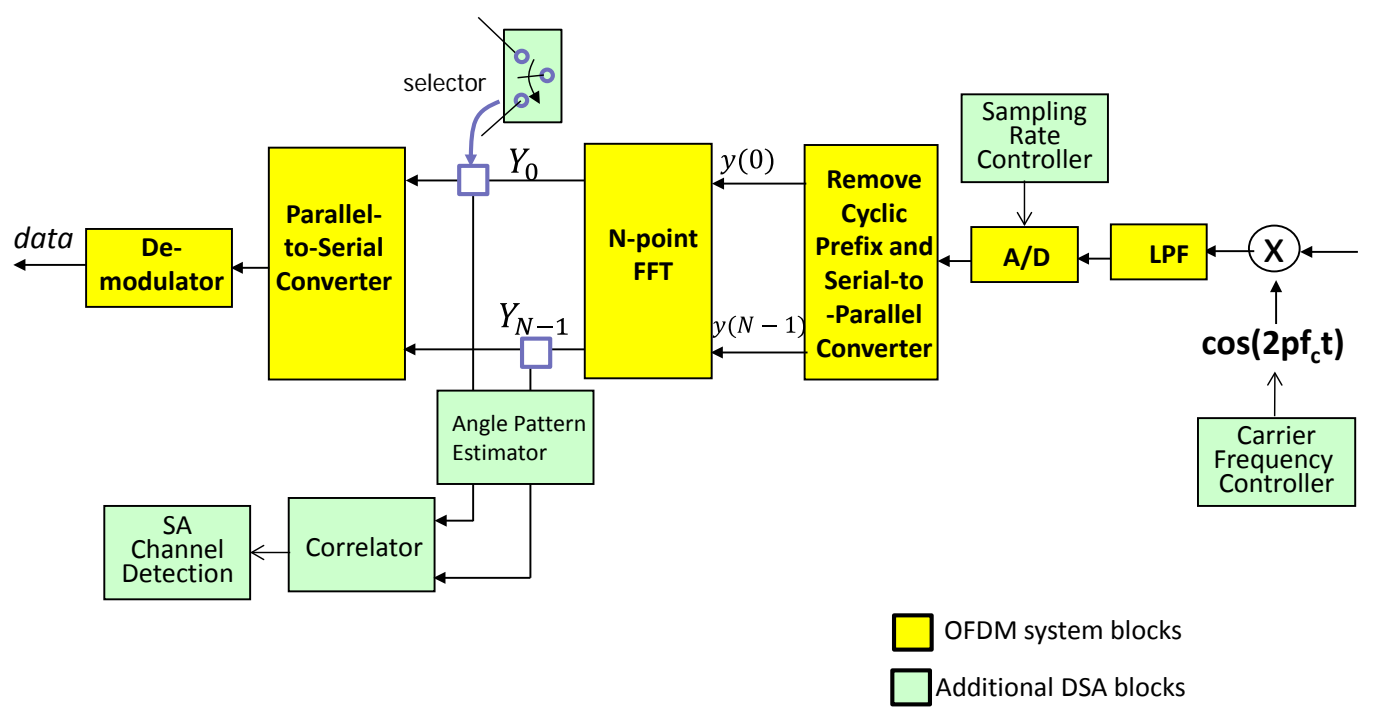

(b) System architecture of the receiver.

Fig. 11. Proposed system architecture.

\section{Experimental Simulation Results}

In this section, we evaluate the performance of the proposed common channel initialization protocol. The simulation parameters are shown in Table 2 . We divided the entire bandwidth into 16 channels, and each channel has two subcarriers (one boundary subcarrier per channel). This means that each channel has only one available activated subcarrier. We assume that eight channels are used by primary systems from among the 16 channels. The actual transmission channels are influenced by the number of subcarriers, because we partially used the available subcarriers. In this simulation, the total number of subcarriers is 32, and the number of available subcarriers is eight. So, the available subcarriers are affected by the signal-to-noise ratio (SNR) among the subcarriers. Therefore, the effective SNR (ESNR) can be defined in (8):

$$
\mathrm{ESNR}=\mathrm{SNR}+10 \log _{10}\left(\frac{\text { Number of subcarrier }}{\text { Number of activated subcarrier }}\right)
$$

Table 2. Simulation parameters

\begin{tabular}{|c|c|}
\hline Parameters & Value \\
\hline \hline SNR $(\mathrm{dB})$ & $0 \sim 10$ \\
\hline Average angle difference of pattern signals & $45,90,135,180$ \\
\hline Pattern length & $16,32,48,64$ \\
\hline Number of available activated subcarriers & $2,4,8,16$ \\
\hline
\end{tabular}

We calculated the matching value (matching rate) for both cases: i) only additive white Gaussian noise (AWGN), and ii) SA signal + AWGN, in which the SA signal and AWGN are combined on each subcarrier. In Fig. 12 and Fig. 13, for the product-based matching method, where SNR $=0 \mathrm{~dB}$ and average angle difference $=135$, we can see that SA signal + AWGN matched the pattern value on a higher matching value because the product-based matching 
method evaluates the matching value in (5). This means that the SA signal + AWGN is better than only AWGN. For example, pattern length $=64$ and PRO matching value $=0.3$ at CDF figure in Fig. 12 and Fig. 13, our system recognizes the SA signal when PRO matching value is bigger than 0.3. In only AWGN case, 95\% signals are recognized as noise. The performance is a little high in SA signal + AWGN case, in terms of pattern length.
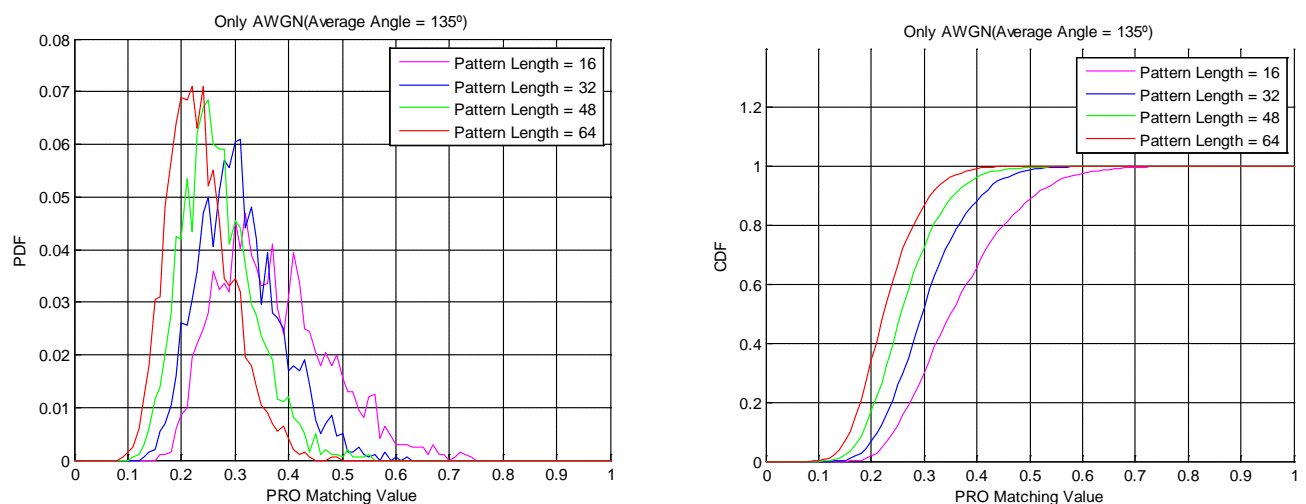

Fig. 12. Matching value for the product-based matching method with only AWGN.
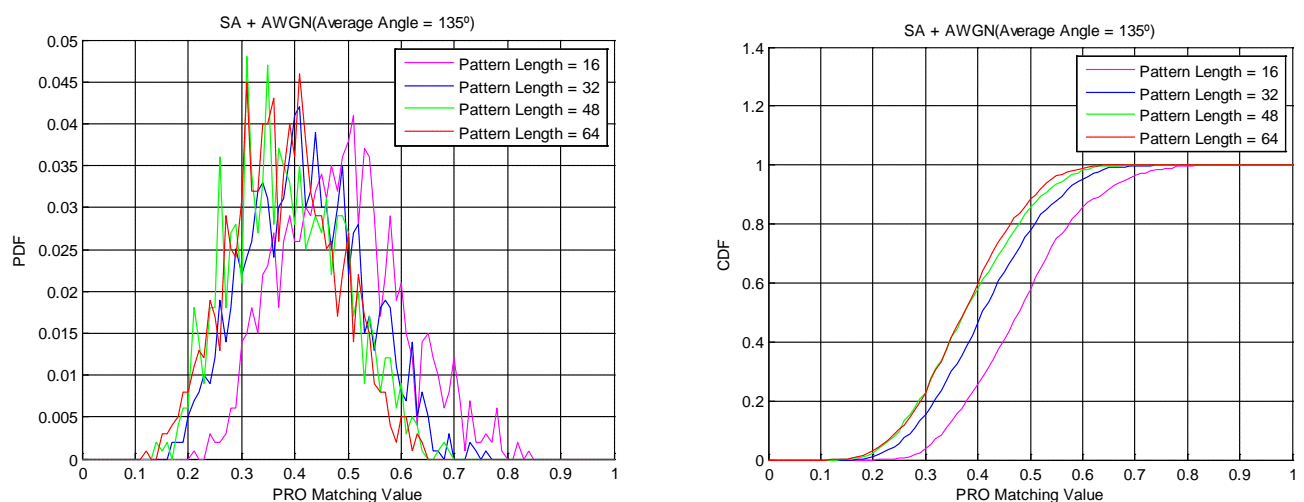

Fig. 13. Matching value for the product-based matching method using SA signal + AWGN.

In Fig. 14 and Fig. 15 for the MSE-based matching method, the lower matching value for SA signal + AWGN indicated a higher possibility of SA signal matching where SNR $=0 \mathrm{~dB}$ and average angle difference $=135$. As we can see in the Fig. 14 and Fig. 15, if the pattern length increases, SA signal detection performance also improves. And MSE method can recognize SA signals 99\% for all pattern length. However, member nodes may take more time to successfully recognize the SA signal with an increase in pattern length for both methods.

Fig. 16 represents the MSE matching value according to the number of available channels. The number of available channels affects ESNR. Fig. 16 shows that if the number of available channels decreases, performance is enhanced due to the reduced interference on each channel. 

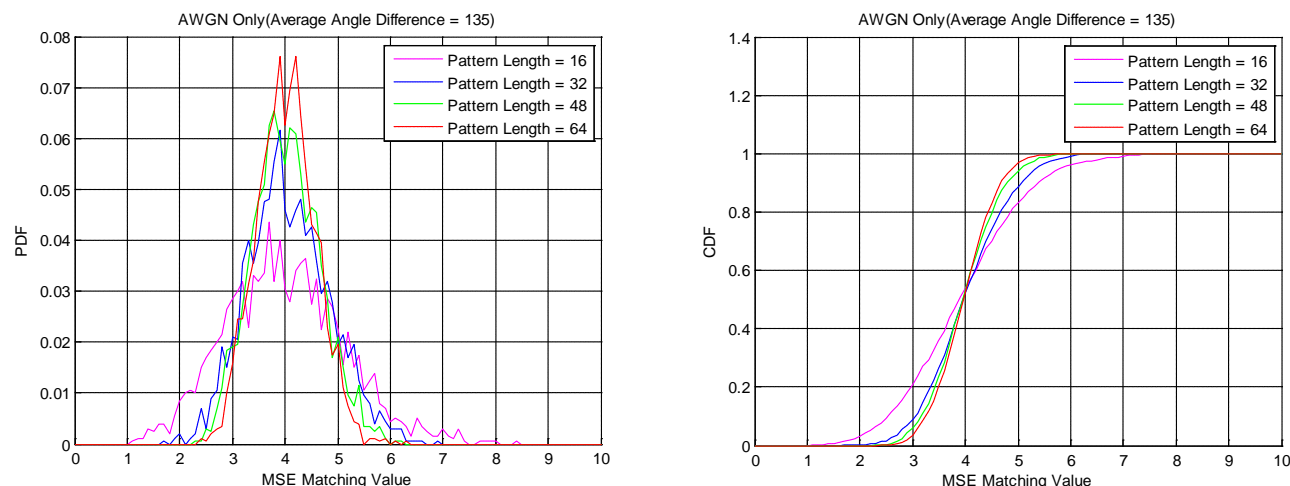

Fig. 14. Matching value for MSE-based matching method with only AWGN.
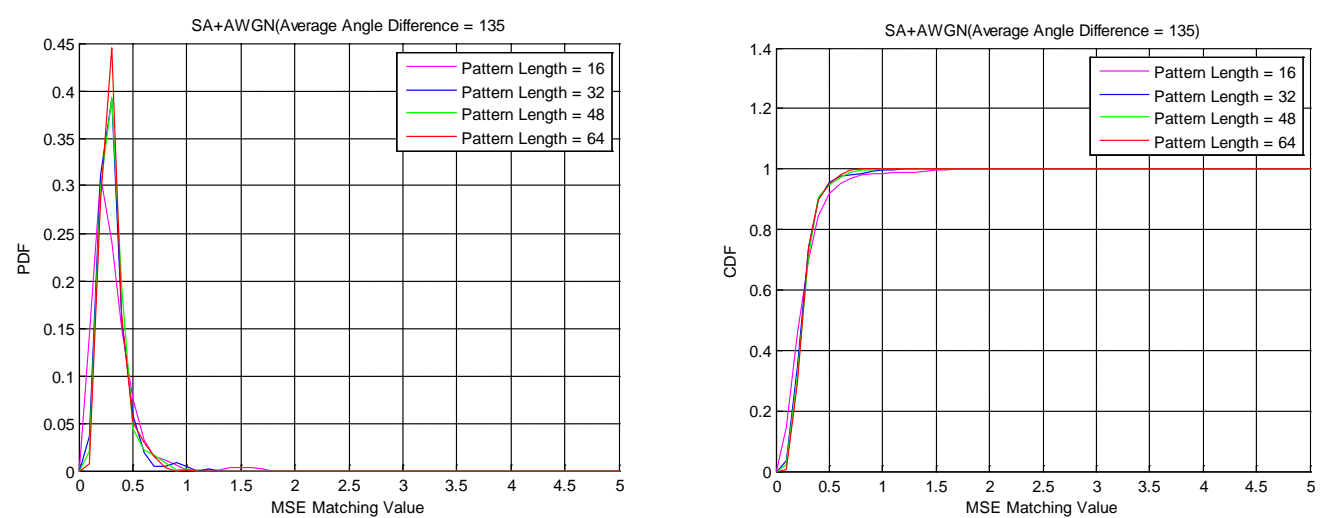

Fig. 15. Matching value for MSE-based matching method using SA signal + AWGN.

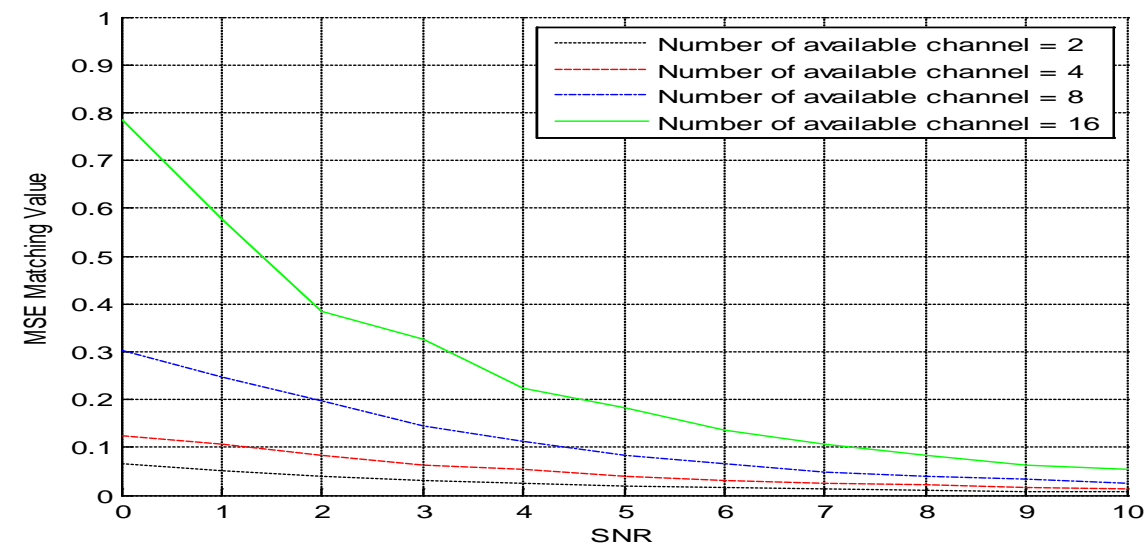

Fig. 16. Matching value for MSE-based matching method according to the number of available channels.

We also calculated the misrecognition ratio (MRR) that indicates the situation where a member node did not recognize the SA pattern on the channel sent by the cluster head. In Fig. 17, we fixed the pattern length to 16 in the product-based matching method. If we set the threshold to more than 0.55 , then it is possible to have less than $5 \%$ of the MRR, except when average angle difference is 45 . It should also be noted that for the same threshold (threshold = 
5.5), the false recognition ratio (FRR), in which an only-AWGN signal can be decoded as the SA signal, is less than $5 \%$ for all average angle signals.

Fig. 18 illustrates FRR and MRR for the MSE-based pattern matching method. Also, if we set the threshold to more than 2.5, then it is possible to have less than $2 \%$ MRR, except when the average angle difference is 45 . It should also be noted that for the same threshold $(=2.5)$, the FRR, which indicates that an only-AWGN signal can be decoded as the SA signal, is less than $2 \%$ for all average angle signals. When average angle difference is 90 and the threshold is higher than 2.5, then MRR is less than 2\%.
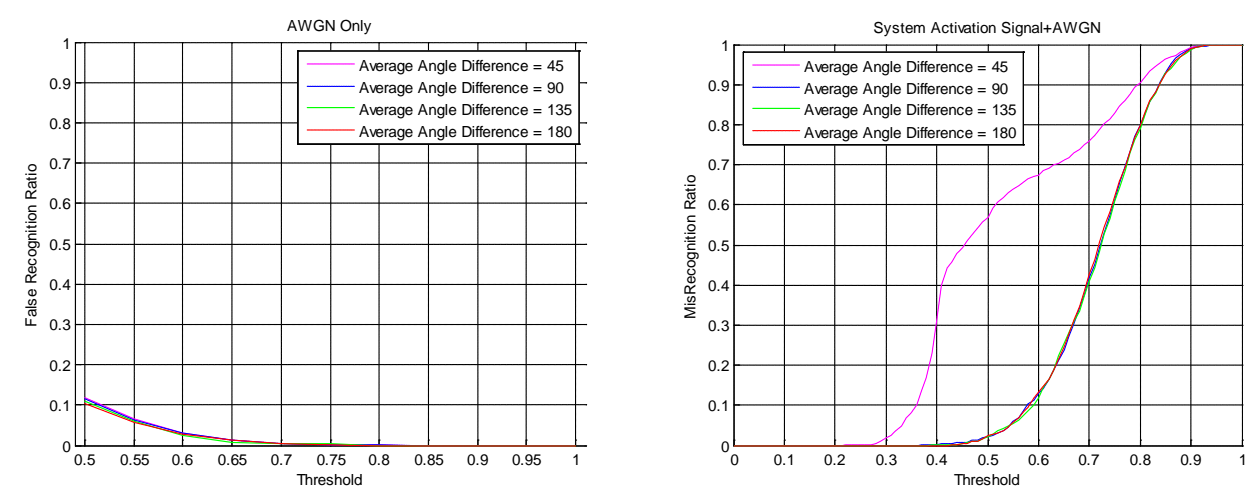

Fig. 17. FRR and MRR for the product-based pattern matching method.
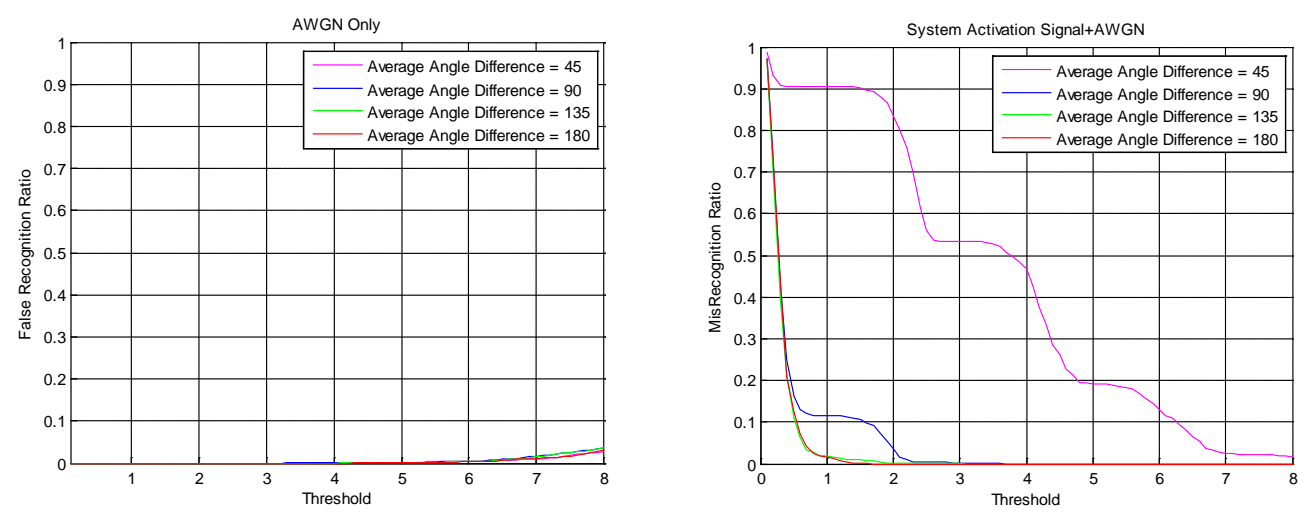

Fig. 18. FRR and MRR for the MSE-based pattern matching method.

Fig. 19 illustrates the misrecognition ratio according to pattern length where average angle difference $=135$ and SNR $=0 \mathrm{~dB}$. In this case, the misrecognition ratio is lower compared to a low threshold and a large threshold. As we can see, if the threshold is larger than 1.5, MRR is less than $2 \%$, except when pattern length $=16$.

Fig. 20 shows the performance comparison for the maximum time to rendezvous (MTTR). ASYNC-ETCH [22] is compared as a conventional method. It is a channel hopping based rendezvous protocol and does not assume that the global clock synchronization mechanism exists. Since most of existing rendezvous protocols do not take into account the signal detection failure condition due to noisy environment, for this performance analysis we have removed redundant signal repetition of the proposed method. As shown in Fig. 20, MTTR (in slot time unit) of the proposed method is a function of the signal pattern length $L$, the number of subcarriers of a channel $M$ and the number of boundary subcarriers $N B$. Since the proposed 
one uses multicarrier operation, we can send signals for multiple channels (and subcarriers) at the same time, MTTR of the proposed method does not increase as increasing the number of channels $(N)$ unlike ASYNC-ETCH case.

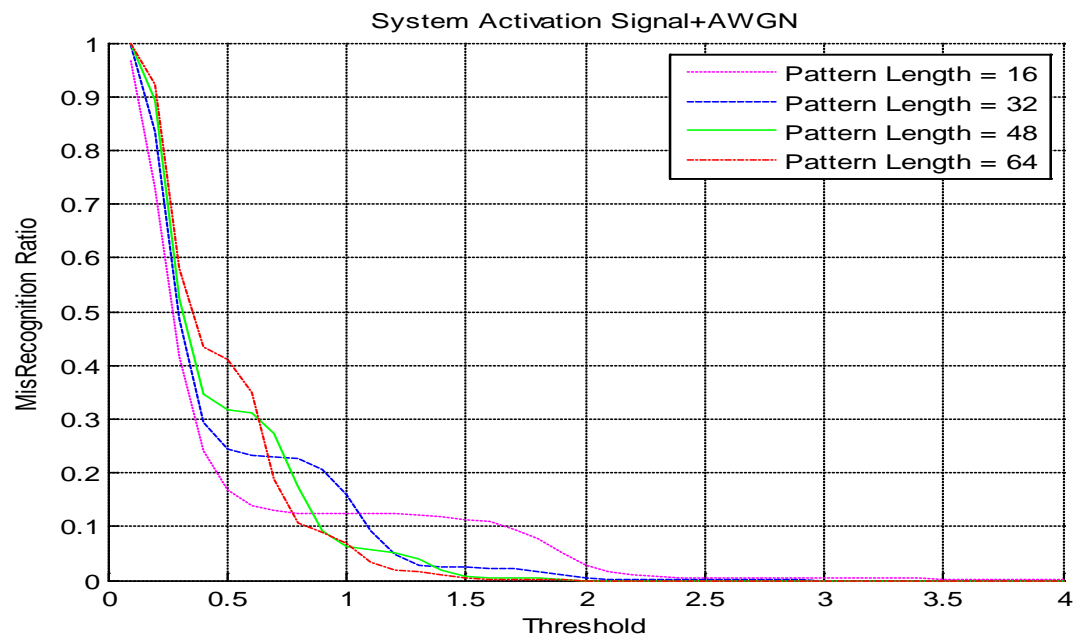

Fig. 19. MRR for the MSE-based pattern matching method according to pattern length.

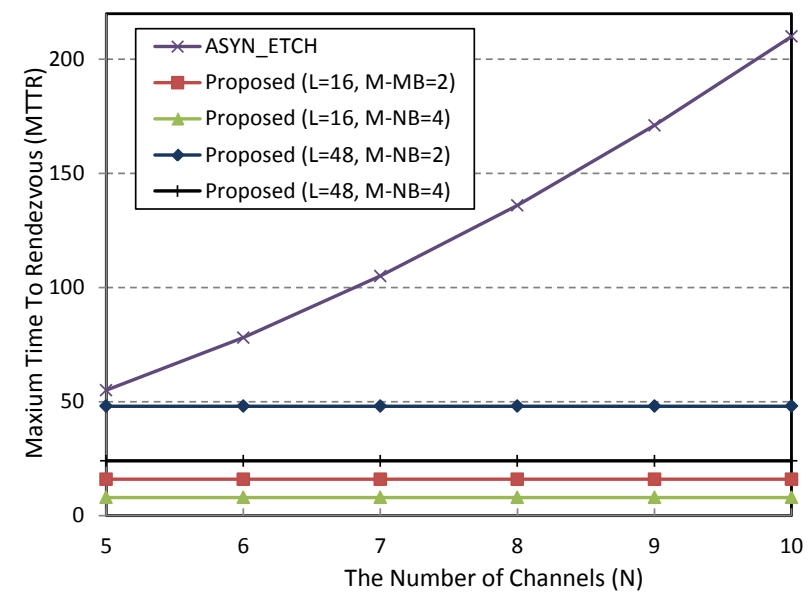

Fig. 20. Maxium time to rendezvous (MTTR) comparison.

\section{Conclusions}

In this paper, we proposed a method for setting a common channel for data communications between secondary users that include a cluster head and member nodes in ad-hoc cognitive radio wireless networks. The cluster head transmits an SA signal, which has an angle difference pattern. Member nodes decode the angle difference pattern signal, which they can easily detect. Initial synchronization is not needed because they can quickly perform symbol boundary detection. We can differentiate clusters by using different angle difference patterns for different groups. Using multi-channel OFDM technology, member nodes can perform SA signal detection. In the simulation results, we confirmed that the proposed pattern matching method showed very low misrecognition and false-recognition ratios under various conditions. 
We should adjust the pattern length and average angle difference, however, because these values have an interrelationship.

\section{References}

[1] "Spectrum Policy Task Force Seeks Public Comment on Issues Related to Commission's Spectrum Policies,” ET Docket No. 02-135, Public Notice (DA 02-1311) (rel. June 6, 2002). Article (CrossRef Link)

[2] J.L. Mauri, K.Z Ghafoor, D.B. Rawat and J. M. Perez, "Cognitive Networks: Applications and Deployment," CRC Press, New York, 2017. Article (CrossRef Link)

[3] J. Choi and S. Yoo " An efficient routing protocol considering path reliability in cognitive radio ad-hoc networks," The Journal of Korea Information and Communications Society, vol. 39B, no. 11, pp. 730-742, Nov. 2014. Article (CrossRef Link)

[4] Saleem Yasir, Rehmani Mubashir Husain "Primary radio user activity models for cognitive radio networks: A survey,” Journal of Network and Computer Applications,” Vol. 431p 16p, 2014. Article (CrossRef Link)

[5] Jiang Dingde, Wang Yuanting, Yao Chunping, Han Yang "An effective dynamic spectrum access algorithm for multi-hoc cognitive wireless networks," Journal Computer Networks: The International Journal of Computer and Telecommunications Networking, Vol. 84 1p 16p, 2015. Article (CrossRef Link)

[6] I. F. Akyildiz, W. Y. Lee, M. C. Vuran, and S. Mohanty, "Next generation dynamic spectrum access cognitive radio wireless networks: a survey," Elsevier Computer Networks, vol. 50, no.13, pp. 2127-2159, 2006. Article (CrossRef Link)

[7] Brandon F. Lo, "A survey of common control channel design in cognitive radio networks, "Phys. Commun. (PHYCOM), vol. 4, no. 1, pp. 26- 39, Mar. 2011. Article (CrossRef Link)

[8] C. Corrnio and K. R. Chowdhury, "An adaptive multiple rendezvous control channel for cognitive radio wireless ad hoc networks," in Proc. of IEEE Int. Conf. Pervasive Comput. Commun. (PERCOM 2010), pp. 346-351, Mannheim, Germany, Apr. 2010. Article (CrossRef Link)

[9] I-Hsun Chuang, Hsiao-Yun Wu, Yau-Hwang Kuo, "A Fast Blind Rendezvous Method by Alternate Hop-and-Wait Channel Hopping in Cognitive Radio Networks", IEEE Transactions on Mobile Computing, Vol. 13, No. 10, pp. 2171 - 2184, 2014. Article (CrossRef Link)

[10] Min-Gyu Kim, Sang-Jo Yoo, Young-Up Jang, Kwang-Eog Lee, "Reliable Cluster-based Common Channel Setup Method for DSA CR Networks," in Proc. of ICUFN 2013, pp. 155-160, 2013. Article (CrossRef Link)

[11] T. Chen, H. Zhang, G. M. Maggio, and I. Chlamtac, "CogMesh: a clusterbased cognitive radio network," in Proc. of ICCC Dyn. Spectr. Access Netw. (DySPAN 2007), pp. 168-178, Dublin, Ireland, Apr. 2007. Article (CrossRef Link)

[12] A. Li, G. Han, J. J. P. C. Rodrigues and S. Chan, "Channel Hopping Protocols for Dynamic Spectrum Management in 5G Technology," IEEE Wireless Communications, Vol. 24, No. 5, pp. 102-109, 2017. Article (CrossRef Link)

[13] G. P. Joshi, S. Y. Nam and S. W. Kim, "Rendezvous Issues in AD Hoc Cognitive Radio Networks," KSII TRANSACTIONS ON INTERNET AND INFORMATION SYSTEM, vol. 8, no. 11, Nov. 2014. Article (CrossRef Link)

[14] K. Bian, J. M. Park and R. Chen, "Control Channel Establishment in Cognitive Radio Networks using Channel Hopping," IEEE Journal on Selected Areas in Communications, Vol. 29, No. 4, pp. 689-703, 2011. Article (CrossRef Link)

[15] I. Chuang, H. Wu, and Y. Kuo, "A Fast Rendezvous-Guarantee Channel Hopping Protocol for Cognitive Radio Networks," IEEE Transactions on Vehicular Technology, Vol. 64, No. 12, pp. 5804-5816, 2015, Article (CrossRef Link)

[16] C. M. Chao and H. Y. Fu, "Supporting Fast Rendezvous Guarantee by Randomized Quorum and Latin Square for Cognitive Radio Networks," IEEE Transactions on Vehicular Technology, Vol. 65, No. 10, pp. 8388-8399, 2016. Article (CrossRef Link) 
[17] J. P. Sheu, C. W. Su and G. Y. Chang, "Asynchronous Quorum-Based Blind Rendezvous Schemes for Cognitive Radio Networks," IEEE Transactions on Communications, Vol. 64, No. 3, pp. 918-930, 2016. Article (CrossRef Link)

[18] C. Chao and H. Fu, "A Fast and Fair Rendezvous Guarantee Channel Hopping Protocol for Cognitive Radio Networks," in Proc. of IEEE International Conference on Communication Software and Networks (ICCSN), Beijing, pp. 483-487, 2016. Article (CrossRef Link)

[19] K. Bian and J. M. Park, "Maximizing Rendezvous Diversity in Rendezvous Protocols for Decentralized Cognitive Radio Networks," IEEE Transactions on Mobile Computing, Vol. 12, No. 7, pp. 1294-1307, 2013. Article (CrossRef Link)

[20] Mi-Ryeong Kim, Sang-Jo Yoo, "Distributed Coordination Protocol for Ad-hoc Cognitive Radio Networks”, Journal of Communications and Networks, Vol.14, No.1, pp. 51-62. 2012. Article (CrossRef Link)

[21] Ik-Soo Choi, Sang-Jo Yoo, Myunghwan Seo, Hyung-Weon Cho, "Initial Rendezvous Protocol Using Multicarrier Operation for Ad-hoc Cognitive Radio Networks,” in Proc. of International Conference on Information and Communication Technology Convergence (ICTC2016), pp. 121-125, Jeju, July, 2016. Article (CrossRef Link)

[22] Y. Zhang, Q. Li, G. Yu and B.Wang, "ETCH: Efficient channel hopping for communication rendezvous in dynamic spectrum access networks," in Proc. of IEEE INFOCOM, pp. 2471-2479, April, 2011. Article (CrossRef Link)

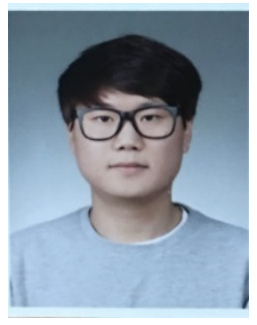

Ik-Soo Choi is currently pursuing a master degree at Inha University. His current research interests include cognitive radio, wireless communications, communication engineering, artificial intelligence.

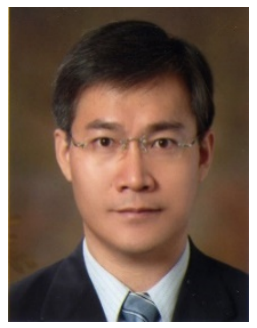

Sang-Jo Yoo received the B.S. degree in electronic communication engineering from Hanyang University, Seoul, Korea, in 1988 and the M.S. and Ph.D. degrees in electrical engineering from Korea Advanced Institute of Science and Technology (KAIST), in 1990 and in 2000, respectively. From 1990 to 2001, he was a member of technical staff at Korea Telecom Research and Development Group, where he worked in communication protocol conformance testing and network design fields. From September 1994 to August 1995 and from January 2007 to January 2008, he was a guest researcher at National Institute Standards and Technology (NIST), USA. Since 2001, he has been with Inha University, where he is currently a professor at Information and Communication Engineering Department. His current research interests include cognitive radio network protocols, seamless network mobility control, wireless network QoS, and wireless sensor networks. 


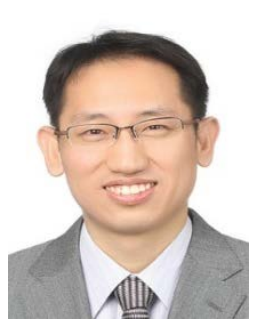

Myunghwan Seo Yoo received the B.S. degree in information and communications engineering from Chungnam National University, Korea, in 2002 and the M.S. and Ph.D. degrees in school of engineering from Information and Communications University (merged with Korea Advanced Institute of Science and Technology), Korea, in 2004 and in 2009, respectively. Currently, he is working at Hanwha System, as a chief engineer. His research interests include cognitive ad-hoc network, military communications systems, and network synchronization.

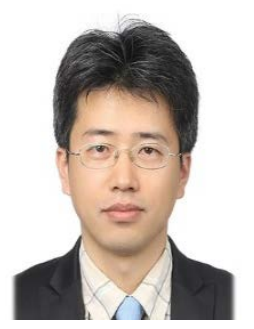

Chulhee Han received the B.S. degree in Electronic Engineering from Chung-ang University, Korea, in 1997, and M.S. and Ph.D. degrees in Electrical and Electronic Engineering from Yonsei University, Korea, in 1999 and 2007, respectively. Currently, he is working at Hanwha Systems, Korea, as a chief engineer. He was involved in various projects including tactical mobile WiMAX system and tactical LOS PMP radio. His research interests include Tactical Broadband Communications, Combat Network Radio, and Cognitive Radio for Military Applications.

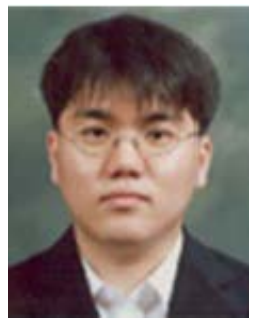

Bongsoo Roh received the B.S. degree in Electrical and Electronic Engineering from Hanyang University, Seoul, Korea, in 2004, and the M.S. degree in Computer Engineering from Pohang University of Science and Technology (POSTECH) in Pohang, Korea, in 2006. Since 2006, he has been with Agency for Defence Development (ADD) in Daejeon, Korea. His research interests include cognitive adhoc network, mobile communications systems, and network performance analysis. 